\title{
Plasma mitochondrial DNA levels are associated with acute lung injury and mortality in septic patients
}

\author{
Jia-yu Mao ${ }^{\dagger}$, Dong-kai Li ${ }^{\dagger}$, Hong-min Zhang, Xiao-ting Wang ${ }^{*}$ and Da-wei Liu*
}

\begin{abstract}
Background: Mitochondrial DNA (mtDNA) is a critical activator of inflammation. Circulating mtDNA released causes lung injury in experimental models. We hypothesized that elevated plasma mtDNA levels are associated with acute lung injury (ALI) in septic patients.

Methods: We enrolled 66 patients with sepsis admitted to the Department of Critical Care Medicine of Peking Union Medical College Hospital between January 2019 and October 2019. Respiratory, hemodynamic and bedside echocardiographic parameters were recorded. Plasma mtDNA, procalcitonin, interleukin 6 , and interleukin 8 levels were examined.

Results: Plasma mtDNA levels within $24 \mathrm{~h}$ after admission were significantly increased in the group of septic patients with ALI [5.01 (3.38-6.64) vs 4.13 (3.20-5.07) log copies/ $\mu \mathrm{L}, \mathrm{p}$ 0.0172]. mtDNA levels were independently associated with mortality (hazard ratio, 3.2052; 95\% Cl 1.1608-8.8500; p 0.0253) and ALI risk (odds ratio 2.7506; 95\% Cl 1.16476.4959; $\mathrm{p}$ 0.0210). Patients with high mtDNA levels had worse outcomes, and post hoc tests showed significant differences in 28-day survival rates. Increased mtDNA levels were seen in patients with abdominal infection.
\end{abstract}

Conclusions: Increased plasma mtDNA levels within $24 \mathrm{~h}$ after admission were significantly associated with ALI incidence and mortality in septic patients.

Keywords: Plasma mtDNA level, Biomarkers, Acute lung injury, Sepsis

\section{Background}

Mitochondrial DNA (mtDNA), as a potential damageassociated molecular pattern (DAMP), has attracted intense research in recent years since it may contribute to the mechanism by which mitochondria regulate innate immunity [1]. Cell stress and necrosis caused by injury may result in fragmentation of the mitochondrial genome and disruption of the mitochondrial membrane, ultimately leading to extracellular release of mitochondrial

\footnotetext{
*Correspondence: icuting@163.com; pumchicuky@163.com

${ }^{\dagger} J i a-y u$ Mao and Dong-kai Li contributed equally to this study and share first authorship

Department of Critical Care Medicine, Peking Union Medical College Hospital, Peking Union Medical College and Chinese Academy of Medical Science, 1 Shuaifuyuan Road, Dongcheng District, Beijing 100730, China
}

DNA $[2,3]$. Circulating mtDNA is capable of triggering innate immunity through multiple mechanisms, such as activating the Toll-like receptor 9 (TLR-9)/NF- $\mathrm{KB}$ pathway or NALP3 inflammasome $[4,5]$. As a consequence, mtDNA may amplify the injury and contribute to the persistent and dysregulated inflammatory response.

Growing evidence has revealed that extracellular mtDNA might act as a biomarker in critically ill patients. Elevated levels of plasma mtDNA are associated with sepsis, trauma or postcardiac arrest [1, 6-9]. Intervention with mtDNA in an animal study induced systemic inflammatory response syndrome (SIRS) and pulmonary edema [4], and blockade of TLR9 improved the lung histopathological changes, wet/dry ratios and inflammatory factor concentrations [10]. mtDNA has been shown 
to increase endothelial cell permeability, either directly or through interactions with endothelial cells and polymorphonuclear leukocytes [11]. Therefore, circulating mtDNA may account for lung injury during nonpulmonary insults, such as sepsis.

In this study, we explored the relationship between circulating mtDNA and septic lung injury, especially at extrapulmonary infection sites. We hypothesized that plasma mtDNA levels may reflect the level of septic organ dysfunction and prognosis.

\section{Methods}

\section{Study design}

We performed a prospective study among patients in the Department of Critical Care Medicine of Peking Union Medical College Hospital (PUMCH) from January 2019 to October 2019, which was approved by the PUMCH institutional review board (approval number JS-1985). All methods were performed in accordance with the relevant guidelines and regulations, informed consent was obtained from all enrolled patients through the next of kin of each patient. The inclusion criteria in this study were as follows: (1) age $\geq 18$ years, (2) intensive care unit (ICU) stay of more than $24 \mathrm{~h}$, and (3) sepsis diagnosis (see below). Patients who were younger than 18 years old, discharged within $24 \mathrm{~h}$ after admitted, or had basic pulmonary disease were excluded.

Sepsis was defined according to consensus international guidelines as life-threatening organ dysfunction caused by a dysregulated host response to infection [12]. Organ dysfunction was defined as an acute change in total Sequential Organ Failure Assessment (SOFA) score of $\geq 2$ points consequent to the infection. Acute lung injury (ALI) was considered to be present if $\mathrm{PaO}_{2} /$ $\mathrm{FiO}_{2}$ (oxygen index, OI) $\leq 300 \mathrm{mmHg}$ [13]. The enrolled patients were divided into a control group composed of patients without ALI and an ALI group. The sample size was estimated based on a priori power calculation indicating an $80 \%$ power to detect a difference of mtDNA copy number frequency (effect size 0.7 ) between control and ALI group at the 0.05 significance level using a power and sample size website (http://powerandsamplesize. com).

\section{Outcome}

Vital sign tests, laboratory tests, echocardiography, analysis of plasma mtDNA copies and clinical history evaluation were carried out on the first day after ICU admission. Basic clinical and laboratory characteristics included age, sex, basic cardiological or pulmonary disease, hemodynamic parameters, respiratory parameters, blood chemistry, arterial blood gas analysis, Acute Physiology and Chronic Health Evaluation (APACHE) II score, and SOFA score. Life-sustaining treatment included mechanical ventilation, vasopressor administration, and renal replacement. Follow-up data included ICU stay time and 28-day mortality rate.

Peripheral blood samples were collected within $24 \mathrm{~h}$ of ICU admission and centrifuged immediately, and the plasma was withdrawn and stored at $-80^{\circ} \mathrm{C}$ until assessment of mtDNA copies. Plasma mtDNA was extracted using a commercially available kit (DP 318, Tiangen Biochemistry, Beijing), quantified using polymerase chain reaction (PCR) for the mitochondrial ND1 gene and measured in triplicate as reported previously. The fold-change was calculated by the $2^{-\Delta \Delta C q}$ method compared with standards in the kit and showed using logtransformed copy number/ $\mu \mathrm{L}$ to ensure normality $[6,14]$. The primers for the mtND1 gene were forward $5^{\prime}$-ATA CCCATGGCCAACCTCCT- $3^{\prime}$ and reverse $5^{\prime}$-GGGCCT TTGCGTAGTTGTAT- $3^{\prime}$.

New onset RV dysfunction was assessed using multimodality parameters as defined by the American Society of Echocardiography (ASE) criteria, i.e., specifically semiquantitative size and function, tricuspid annular plane systolic excursion (TAPSE) $<16 \mathrm{~mm}$ by M-mode, tricuspid lateral annulus tissue Doppler systolic velocity $<0.15 \mathrm{~cm} / \mathrm{s}$ or RV fractional area change $<35 \%$ [15]. Echocardiographic examinations were performed by two experienced intensivists. An ultrasonic machine (M-Turbo Sonosite, Highland Heights, Ohio) equipped with a $15 \mathrm{MHz}$ transducer was used for noninvasive transthoracic echocardiography. Data from 3 consecutive cardiac cycles were analyzed and averaged.

\section{Statistical analysis}

The normality of the data was evaluated using the Kolmogorov-Smirnov test. Normally distributed data were expressed as the mean and standard deviation and were compared using Student's t-test. Nonnormally distributed data are presented as the median, and interquartile intervals were analyzed with the Mann-Whitney U test. Categorical variables were recorded as proportions. Univariate logistic regression was performed to identify mtDNA and other parameters with predictive value for 28-day mortality and ALI risk. The Cox proportional hazard survival model was applied to identify the independent contribution of prognostic factors to the prediction of the 28-day outcome. The results are expressed as the Wald index, odds ratio (OR), hazard ratio (HR) and 95\% CIs. Discrimination of values was calculated using receiver operating characteristic (ROC) curve analysis using the Hanley-McNeil test. Survival curves up to day 28 were estimated using the Kaplan-Meier method, and the log-rank (Mantel-Cox) test was used to estimate differences among the predefined groups. All comparisons 
were two-tailed, and a p value less than 0.05 was required to exclude the null hypothesis. Statistical analyses were performed using the SPSS 13.0 software package (SPSS, Chicago, IL).

\section{Results}

\section{Basic characteristics}

During the study period (from January to October 2019), 101 patients with sepsis were admitted to the Critical Care Medicine Department of PUMCH. Of these, 32 satisfied at least 1 of the exclusion criteria ( 3 were $<18$ years old, 21 were discharged with $24 \mathrm{~h}$ after admission, and 8 had basic pulmonary disease), and 3 patients refused to sign the consent form. A total of 66 patients were enrolled in the study, 40 of whom met the ALI criteria.

The basic clinical characteristics of all patients included in this study after ICU admission are shown in Table 1. The majority of patients were male, and elderly individuals accounted for a large portion of the ALI patients. The composition of pulmonary infection sources was accordant in patients with or without lung injury; however, bloodstream and abdominal infections seemed to be more common in patients with ALI. The mortality, ICU stay time and APACHE II and SOFA scores were significantly higher in the ALI group than in the control group. In terms of respiratory condition, the OI, plat pressure (Pplat), and positive end expiratory pressure (PEEP) were worse in the ALI group. Regarding hemodynamic data, compared with those in the control group, patients with ALI had a higher central venous pressure (CVP), and no significant difference in mean arterial pressure (MAP), heart rate (HR) or norepinephrine (NE) dose was seen. Only mtDNA [5.01 (3.38-6.64) vs 4.13 (3.20-5.07), p 0.0172 ] (Fig. 1a) as a biomarker rather than procalcitonin $(\mathrm{PCT}), \mathrm{C}$-reactive protein (CRP) or other inflammatory factors, such as interleukin 6 (IL-6), interleukin 8 (IL-8), interleukin 10 (IL-10) or tumor necrosis factor $\alpha$ (TNF $\alpha$ ), showed significant differences between the different groups.

\section{Risk factors for mortality in sepsis patients}

A univariate logistic regression analysis indicated that variables including mtDNA level, APACHE II score, SOFA score, OI, PEEP, CVP and NE level were associated with mortality in septic patients. Cox proportional hazard survival model was used then to adjust relevant variables to examine possible risk factors for a poor prognosis. The HR of mtDNA after adjusted was 3.2052 (95\% CI 1.1608-8.8500; $\mathrm{p}$ 0.0253) (Tables 2, 3).

\section{The mtDNA level was associated with mortality}

The ROC curve was drawn for mortality (Fig. 2a). The area under the curve for mtDNA was 0.856 (95\% CI
Table 1 The general characteristics of the patients included in this study

\begin{tabular}{|c|c|c|c|}
\hline Characteristics & Control, $n=26$ & ALI, $n=40$ & $p$ \\
\hline Age, years & $49.7(31.8-67.7)$ & $65.6(51.7-79.4)$ & $0.0002^{* * *}$ \\
\hline \multicolumn{4}{|l|}{ Sex, n (\%) } \\
\hline Male & $14(53.8)$ & $28(70.0)$ & \\
\hline \multicolumn{4}{|l|}{ Infection site, n (\%) } \\
\hline Blood & $3(11.5)$ & $11(27.5)$ & \\
\hline Lung & $10(38.5)$ & $14(35.0)$ & \\
\hline Abdomen & $4(15.4)$ & $10(25.0)$ & \\
\hline Other & $9(34.6)$ & $5(12.5)$ & \\
\hline Mortality, n (\%) & $0(0.0)$ & $12(30.0)$ & \\
\hline $\begin{array}{l}\text { Ventilator-free time, } \\
\text { days }\end{array}$ & $3.1(0.3-5.9)$ & $17.9(1.9-34.0)$ & $<0.0001^{* * *}$ \\
\hline ICU stay time, days & $4.5(3.0-6.8)$ & $15.5(8.5-33.5)$ & $<0.0001^{* * *}$ \\
\hline APACHE II & 15.7 (7.7-23.6) & $22.5(12.0-33.0)$ & $0.0069^{* *}$ \\
\hline SOFA & $6.0(3.4-8.6)$ & $9.5(6.0-13.1)$ & $<0.0001^{* * *}$ \\
\hline $\mathrm{Ol}, \mathrm{mmHg}$ & $374(329-420)$ & $220(170-270)$ & $<0.0001^{* * *}$ \\
\hline PEEP, $\mathrm{cmH} 2 \mathrm{O}$ & $5.2(4.7-5.7)$ & $7.0(5.0-9.0)$ & $<0.0001^{* * *}$ \\
\hline Pplat, $\mathrm{cmH} 2 \mathrm{O}$ & $18.0(15.2-20.9)$ & $20.5(16.0-25.0)$ & $0.0226^{*}$ \\
\hline $\mathrm{MAP}, \mathrm{mmHg}$ & $81.3(74.3-88.2)$ & $84.3(74.5-94.1)$ & 0.1835 \\
\hline $\mathrm{HR}$, bpm & $94.2(78.2-110.1)$ & $94.9(74.2-115.5)$ & 0.8861 \\
\hline $\mathrm{CVP}, \mathrm{mmHg}$ & $8.4(6.9-10.0)$ & $9.8(7.9-11.8)$ & $0.0063^{* *}$ \\
\hline $\mathrm{NE}, \mu \mathrm{g} / \mathrm{kg} / \mathrm{min}$ & $0.19(0.02-0.29)$ & $0.18(0.08-0.38)$ & 0.0986 \\
\hline Lactate, $\mathrm{mmol} / \mathrm{L}$ & $2.1(0.8-3.4)$ & $2.2(0.3-4.2)$ & 0.7388 \\
\hline $\begin{array}{l}\text { mtDNA, log copies/ } \\
\mu \mathrm{L}\end{array}$ & $4.13(3.20-5.07)$ & $5.01(3.38-6.64)$ & $0.0172^{*}$ \\
\hline $\mathrm{PCT}, \mathrm{ng} / \mathrm{mL}$ & $7.0(3.8-19.0)$ & $5.9(2.0-20.3)$ & 0.4274 \\
\hline CRP, mg/L & $127(79-175)$ & $125(72-177)$ & 0.8537 \\
\hline IL-6, pg/mL & $139(49-376)$ & $93(28-145)$ & 0.8147 \\
\hline IL-8, pg/mL & $157(42-274)$ & $131(53-247)$ & 0.1971 \\
\hline IL-10, pg/mL & $12(6-21)$ & $8(5-14)$ & 0.2418 \\
\hline TNFa, pg/mL & $13(9-19)$ & $21(13-30)$ & 0.8210 \\
\hline
\end{tabular}

ALI acute lung injury, ICU intensive care unit, APACHE Acute Physiology and Chronic Health Evaluation, SOFA Sequential Organ Failure Assessment, OI oxygen index, PEEP positive end expiratory pressure, Pplat plat pressure, $M A P$ mean arterial pressure, $H R$ heart rate, $C V P$ central venous pressure, $N E$ norepinephrine, $P C T$ procalcitonin, CRP C-reactin protein, IL-6 interleukin 6, IL-8 interleukin 8, IL-10 interleukin 10, TNFa tumor necrosis factor a

$0.748-0.930, \mathrm{p}<0.0001)$. The cutoff value for mtDNA was equal to $5.4169 \log$ copies $/ \mu \mathrm{L}$, based on the maximum Youden index. Based on the mtDNA cutoff, all the patients were divided into a low mtDNA group and a high mtDNA group. Post hoc tests showed significant differences in 28-day survival rates between the different groups [log-rank (Mantel-Cox), 6.842; p 0.0089] (Fig. 2b).

The clinical characteristics of patients with different mtDNA amounts are shown in Table 4. There were no significant differences in age, sex, or underlying cardiac disease between the different groups. The mortality $(50 \%$ vs $2.3 \%)$, ICU stay time [21.5 (11.3-42.8) vs $6.0(4.0-12.0)$ 

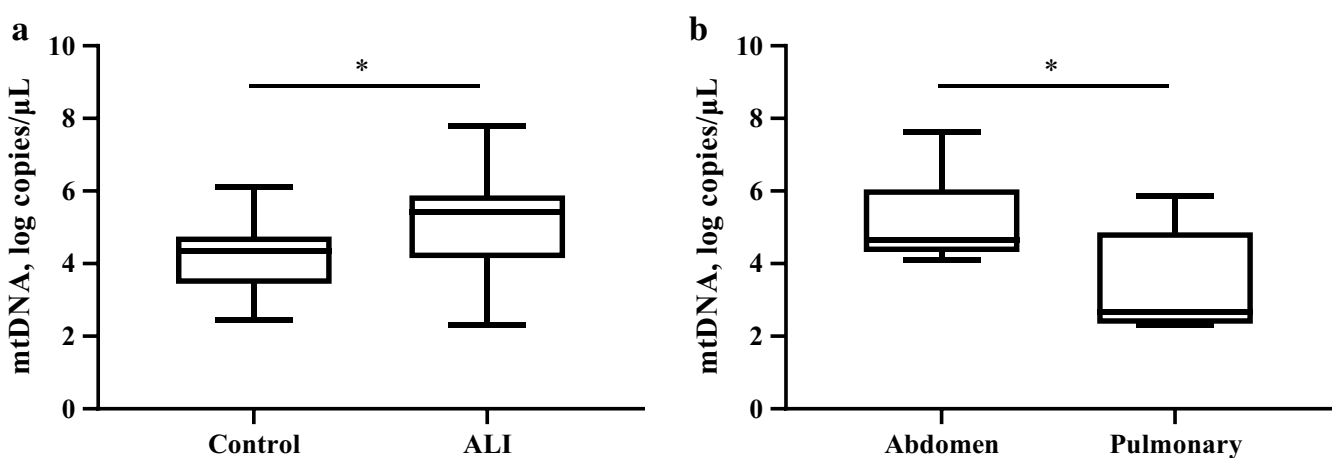

Fig. 1 Comparison of mtDNA level in different groups. a MtDNA level in septic patients with or without acute lung injury. b MtDNA level in septic ALI patients with different infectious sources. ALlacute lung injury. ${ }^{*} \mathrm{p}<0.05$

Table 2 Univariate logistic regression analysis for possible risk factors for prognosis

\begin{tabular}{|c|c|c|c|c|c|c|c|}
\hline \multirow[t]{2}{*}{ Variable } & \multirow[t]{2}{*}{ B } & \multirow[t]{2}{*}{ SE } & \multirow[t]{2}{*}{ Wald } & \multirow[t]{2}{*}{$\mathrm{p}$} & \multirow[t]{2}{*}{ OR } & \multicolumn{2}{|c|}{$95 \% \mathrm{Cl}$ for OR } \\
\hline & & & & & & Lower & Upper \\
\hline Age & 0.0408 & 0.0224 & 3.9240 & 0.0681 & 1.0417 & 0.9970 & 1.0884 \\
\hline APACHE ॥ & 0.0975 & 0.0339 & 9.9070 & $0.0040^{* *}$ & 1.1024 & 1.0315 & 1.1780 \\
\hline SOFA & 0.2714 & 0.1039 & 8.2880 & $0.0090^{* *}$ & 1.3118 & 1.0701 & 1.6080 \\
\hline mtDNA & 1.4154 & 0.4259 & 21.5720 & $0.0009^{* * *}$ & 4.1182 & 1.7872 & 9.4891 \\
\hline Ol & -0.0148 & 0.0054 & 10.8110 & $0.0063^{* *}$ & 0.9853 & 0.9750 & 0.9958 \\
\hline PEEP & 0.5187 & 0.1807 & 9.6210 & $0.0041^{* *}$ & 1.6799 & 1.1789 & 2.3936 \\
\hline Pplat & 0.1385 & 0.0749 & 3.4870 & 0.0642 & 1.1486 & 0.9918 & 1.3301 \\
\hline CVP & 0.3689 & 0.1819 & 4.4290 & $0.0425^{*}$ & 1.4461 & 1.0125 & 2.0653 \\
\hline$N E$ & 3.7879 & 1.1700 & 14.8870 & $0.0012^{* *}$ & 44.1655 & 4.4579 & 437.5610 \\
\hline РCT & 0.0184 & 0.0099 & 3.243 & 0.0643 & 1.0185 & 0.9989 & 1.0386 \\
\hline
\end{tabular}

Table 3 Cox regression analysis for possible risk factors for prognosis

\begin{tabular}{|c|c|c|c|c|c|c|}
\hline \multirow[t]{2}{*}{ Variable } & \multirow[t]{2}{*}{ B } & \multirow[t]{2}{*}{ SE } & \multirow[t]{2}{*}{$p$} & \multirow[t]{2}{*}{ HR } & \multicolumn{2}{|c|}{$95 \% \mathrm{Cl}$ for OR } \\
\hline & & & & & Lower & Upper \\
\hline SOFA & 0.3348 & 0.1675 & $0.0457^{*}$ & 1.3976 & 1.0081 & 1.9377 \\
\hline mtDNA & 1.1648 & 0.5208 & $0.0253^{*}$ & 3.2052 & 1.1608 & 8.8500 \\
\hline Ol & 0.0116 & 0.0077 & 0.1343 & 1.0116 & 0.9965 & 1.0270 \\
\hline PEEP & -0.1750 & 0.2217 & 0.4300 & 0.8395 & 0.5448 & 1.2935 \\
\hline CVP & -0.7199 & 0.3540 & $0.0420^{*}$ & 0.4868 & 0.2441 & 0.9709 \\
\hline $\mathrm{NE}$ & 1.7602 & 0.8862 & $0.0470^{*}$ & 5.8138 & 1.0326 & 32.7319 \\
\hline
\end{tabular}

APACHE Acute Physiology and Chronic Health Evaluation, SOFA Sequential Organ Failure Assessment, Ol oxygen index, PEEP positive end expiratory pressure, CVP central venous pressure, $N E$ norepinephrine

days, $\mathrm{p}<0.0001]$, APACHE II score [29.0 (20.0-38.0) vs $15.2(8.1-22.3), \mathrm{p}<0.0001]$ and SOFA score [10.9 (7.6$14.2)$ vs 6.8 (3.8-9.7), $\mathrm{p}<0.0001$ ] were significantly higher in the group of patients with high mtDNA than in the patients with low mtDNA. Both respiratory and hemodynamic conditions worsened in the high mtDNA group. In terms of respiratory condition, the OI [222 (156-289) vs 339 (255-422) mmHg, p 0.0002], PEEP [7.4 (5.29.7) vs $5.8(4.6-7.0) \mathrm{cmH} 2 \mathrm{O}, \mathrm{p} 0.0005]$, and Pplat [21.5 $(16.8-26.1)$ vs $18.6(15.2-22.0) \mathrm{cmH} 2 \mathrm{O}, \mathrm{p}$ 0.0080] were significantly worse in the high mtDNA group. Regarding hemodynamic data, compared with the control group patients, patients with high mtDNA had a higher lactate value [3.0 (0.6-5.4) vs $1.8(0.7-2.8) \mathrm{mmol} / \mathrm{L}, \mathrm{p} 0.0051]$, 


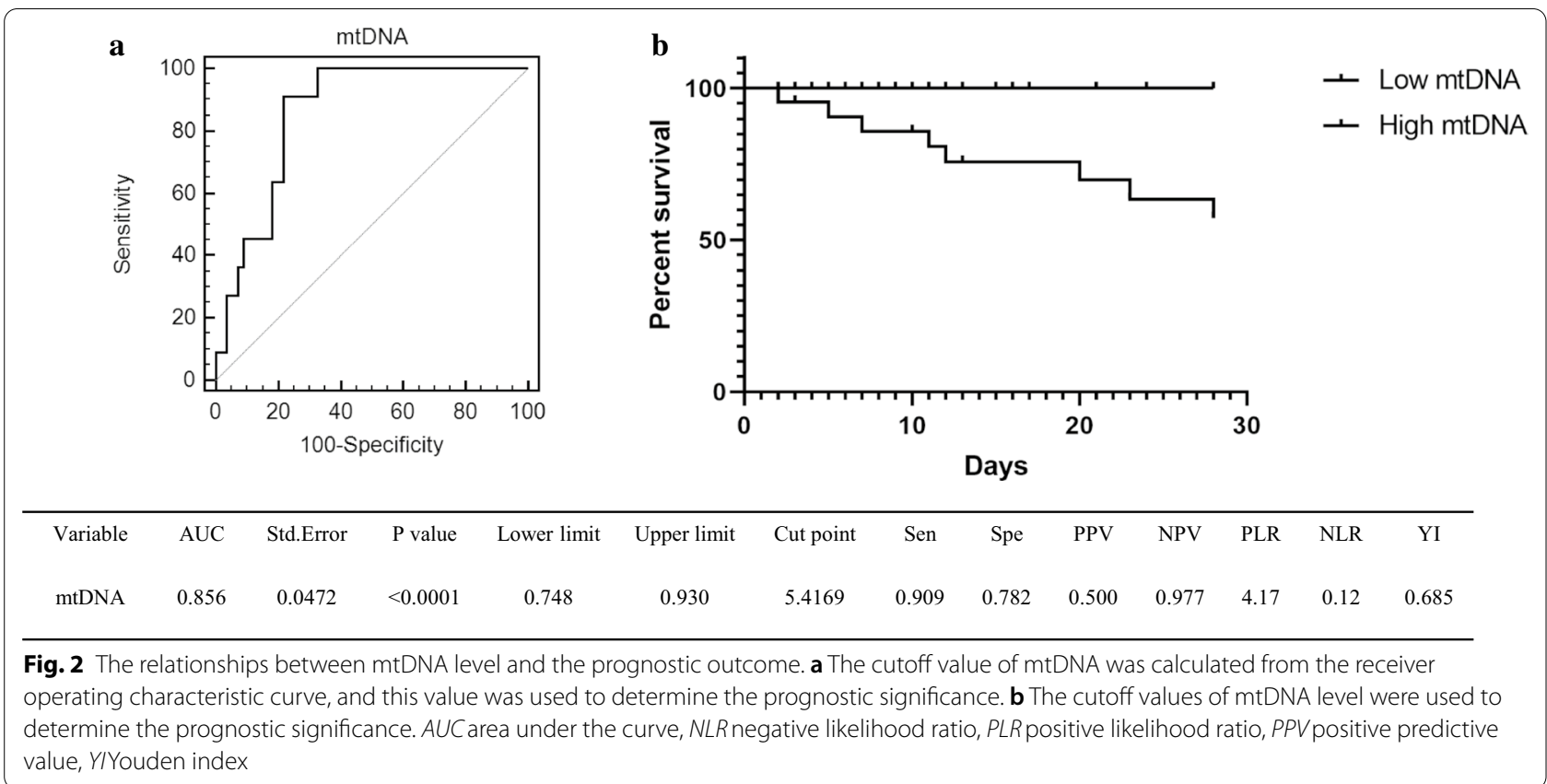

CVP [10.4 (8.8-12.0) vs 8.4 (6.9-9.9) mmHg, p 0.0005], NE dose $[0.27(0.15-0.87)$ vs $0.17(0-0.28) \mu \mathrm{g} / \mathrm{kg} / \mathrm{min}$, p 0.0006], and RV dysfunction occurrence $(59.1 \%$ vs $15.9 \%)$. Levels of other inflammatory biomarkers, such as PCT [7.6 (2.5-52.5) vs $6.0(2.3-19.0) \mathrm{ng} / \mathrm{mL}, \mathrm{p} 0.0091]$, IL-6 [130 (48-966) vs 84 (36-271) pg/mL, p 0.0280], and IL-8 [186 (58-458) vs 131 (42-222) pg/mL, p 0.0121], showed significant differences in patients with different mtDNA amounts.

\section{mtDNA was associated with ALI risk in sepsis patients}

A univariate logistic regression analysis was used to examine ALI risk in sepsis patients. The variables mtDNA, age, APACHE II score, SOFA score, Pplat and PEEP were indicated to be associated with ALI risk in septic patients. A multivariate logistic regression analysis indicated that, the OR of mtDNA after adjusted was 2.7506 (95\% CI 1.1647-6.4959, p 0.0210) (Tables 5, 6).

\section{Comparison of mtDNA in ALI patients with different infectious sources}

Patients with different infectious sources also showed different clinical characteristics (Table 7). No difference was shown in age, sex, ICU stay time, APACHE II and SOFA scores between patients with abdominal or pulmonary infection. However, the OI of abdominal infection patients was even worse than that of pulmonary infection patients [215 (178-243) vs $256(230-271) \mathrm{mmHg}$, p 0.0369]. Patients with abdominal infection showed higher HR [100.0 (95.0-104.5) vs 84.0 (70.0-91.0) bpm, p 0.0176] and NE dose [0.23 (0.06-0.73) vs $0.11(0.05-$ $0.18) \mu \mathrm{g} / \mathrm{kg} / \mathrm{min}, \mathrm{p}$ 0.0427] than patients with pulmonary infection. Higher amounts of mtDNA (Fig. 1b) and other inflammatory biomarkers were seen in patients with abdominal infection [mtDNA, 5.14 (4.39-5.87) vs 4.79 (2.52-5.54) log copies $/ \mu \mathrm{L}, \mathrm{p}$ 0.0492; PCT, 8.1 (3.2-93.3) vs $2.0(0.8-6.5) \mathrm{ng} / \mathrm{mL}, \mathrm{p} 0.0116$; IL-6, 572 (93-1000) vs 38 (17-120) pg/mL, p 0.0006].

\section{Discussion}

To explore the relationship between circulating mtDNA and septic lung injury, we investigated the level of plasma mtDNA within $24 \mathrm{~h}$ after admission in different septic patient groups. We revealed that higher plasma mtDNA levels were associated with ALI incidence and mortality in septic patients. The association was stronger in patients with abdominal infection than in those with pulmonary infection.

Mounting evidence has revealed that extracellular mtDNA might be useful as a biomarker in critically ill patients and is associated with disease severity and mortality [16-18]. Circulating mtDNA may contribute to the mechanism linking distant injury to subsequent lung injury. An increasing number of studies have found elevated mtDNA levels in hip fracture patients with lung injury $[19,20]$, an acute respiratory distress syndrome (ARDS) population from a mixed ICU unit [6] and a cohort of trauma patients [21]. In our study, we further explored whether higher plasma mtDNA levels were associated with ALI incidence and mortality in 
Table 4 Laboratory data parameters in the different groups

\begin{tabular}{|c|c|c|c|}
\hline Characteristics & Low mtDNA, $n=44$ & High mtDNA, $n=22$ & $\mathrm{p}$ \\
\hline Age, years & $57.1(39.3-88.3)$ & $63.8(48.5-79.2)$ & 0.1436 \\
\hline \multicolumn{4}{|l|}{ Sex, n (\%) } \\
\hline Male & $28(63.6)$ & $13(59.1)$ & \\
\hline \multicolumn{4}{|l|}{ Infection site } \\
\hline Blood & $2(4.5)$ & $12(54.5)$ & \\
\hline Lung & $20(45.5)$ & $4(18.2)$ & \\
\hline Abdomen & $9(20.5)$ & $5(22.7)$ & \\
\hline Other & $13(29.5)$ & $1(4.6)$ & \\
\hline Mortality, n (\%) & $1(2.3)$ & $11(50)$ & \\
\hline Ventilator-free time, days & $6.0(1.8-6.7)$ & $22.3(8.0-33.0)$ & $<0.0001^{* * *}$ \\
\hline ICU stay time, days & $6.0(4.0-12.0)$ & $21.5(11.3-42.8)$ & $<0.0001^{* * *}$ \\
\hline RV dysfunction & $7(15.9)$ & $13(59.1)$ & \\
\hline APACHE ॥ & $15.2(8.1-22.3)$ & $29.0(20.0-38.0)$ & $<0.0001^{* * *}$ \\
\hline SOFA & $6.8(3.8-9.7)$ & $10.9(7.6-14.2)$ & $<0.0001^{* * *}$ \\
\hline $\mathrm{Ol}, \mathrm{mmHg}$ & $339(255-422)$ & $222(156-289)$ & $0.0002^{* * *}$ \\
\hline PEEP, $\mathrm{cmH} 2 \mathrm{O}$ & $5.8(4.6-7.0)$ & $7.4(5.2-9.7)$ & $0.0005^{* * *}$ \\
\hline Pplat, $\mathrm{cmH} 2 \mathrm{O}$ & $18.6(15.2-22.0)$ & $21.5(16.8-26.1)$ & $0.0080^{* *}$ \\
\hline MAP, mmHg & $83.4(75.3-91.6)$ & $82.4(72.2-92.7)$ & 0.6809 \\
\hline $\mathrm{HR}$, bpm & $92.0(76.2-107.8)$ & $99.7(76.5-122.9)$ & 0.1216 \\
\hline $\mathrm{CVP}, \mathrm{mmHg}$ & $8.4(6.9-9.9)$ & $10.4(8.8-12.0)$ & $0.0005^{* * *}$ \\
\hline $\mathrm{NE}, \mu \mathrm{g} / \mathrm{kg} / \mathrm{min}$ & $0.17(0-0.28)$ & $0.27(0.15-0.87)$ & $0.0006^{* * *}$ \\
\hline Lactate, $\mathrm{mmol} / \mathrm{L}$ & $1.8(0.7-2.8)$ & $3.0(0.6-5.4)$ & $0.0051^{* *}$ \\
\hline $\mathrm{PCT}, \mathrm{ng} / \mathrm{mL}$ & $6.0(2.3-19.0)$ & $7.6(2.5-52.5)$ & $0.0091^{* *}$ \\
\hline IL-6, pg/mL & $84(36-271)$ & 130 (48-966) & $0.0280^{*}$ \\
\hline IL-8, pg/mL & $131(42-222)$ & $186(58-458)$ & $0.0121^{*}$ \\
\hline IL-10, pg/mL & $8(5-15)$ & $12(6-47)$ & 0.2038 \\
\hline TNFa, pg/mL & $15(9-22)$ & $26(18-36)$ & 0.5511 \\
\hline
\end{tabular}

ICU intensive care unit, $R V$ right ventricular, APACHE Acute Physiology and Chronic Health Evaluation, SOFA Sequential Organ Failure Assessment, OI oxygen index, $P E E P$ positive end expiratory pressure, $P$ plat plat pressure, $M A P$ mean arterial pressure, $H R$ heart rate, $C V P$ central venous pressure, $N E$ norepinephrine, $P C T$ procalcitonin, CRP C-reactin protein, IL-6 interleukin 6, IL-8 interleukin 8, IL-10 interleukin 10, TNFa tumor necrosis factor a

Table 5 Univariate logistic regression analysis for possible risk factors for ALI

\begin{tabular}{|c|c|c|c|c|c|c|}
\hline \multirow[t]{2}{*}{ Variable } & \multirow[t]{2}{*}{ B } & \multirow[t]{2}{*}{ SE } & \multirow[t]{2}{*}{$p$} & \multirow[t]{2}{*}{ OR } & \multicolumn{2}{|c|}{$95 \% \mathrm{Cl}$ for OR } \\
\hline & & & & & Lower & Upper \\
\hline Age & 0.0461 & 0.0171 & $0.0070^{* *}$ & 1.0472 & 1.0127 & 1.0828 \\
\hline APACHE II & 0.1848 & 0.0517 & $0.0003^{* * *}$ & 1.2030 & 1.0871 & 1.3313 \\
\hline SOFA & 0.4558 & 0.1203 & $0.0002^{* * *}$ & 1.5774 & 1.2461 & 1.9970 \\
\hline mtDNA & 0.9020 & 0.2457 & $0.0002^{* * *}$ & 2.4644 & 1.5226 & 3.9889 \\
\hline PEEP & 0.7010 & 0.2578 & $0.0065^{* *}$ & 2.0158 & 1.2161 & 3.3411 \\
\hline Pplat & 0.1629 & 0.0787 & $0.0385^{*}$ & 1.1769 & 1.0086 & 1.3732 \\
\hline
\end{tabular}

APACHE Acute Physiology and Chronic Health Evaluation, SOFA Sequential Organ Failure Assessment, OI oxygen index, PEEP positive end expiratory pressure, CVP central venous pressure, $N E$ norepinephrine

septic patients. Plasma mtDNA levels were significantly increased in the group of septic patients with ALI compared with those in the control group patients [5.01 (3.38-6.64) vs 4.13 (3.20-5.07) log copies/ $\mu \mathrm{L}, \mathrm{p}$ 0.0172]. The sensitivity of other inflammatory biomarkers, such as procalcitonin, CRP IL-6, IL-8, IL-10 and TNF $\alpha$, was inferior to mtDNA among septic.

Consistent with a previous study, we revealed that the mtDNA level (HR, 3.2052; 95\% CI 1.1608-8.8500; p 0.0253 ) was independently associated with mortality, 
Table 6 Multivariate logistic regression analysis for possible risk factors for ALI

\begin{tabular}{|c|c|c|c|c|c|c|}
\hline \multirow[t]{2}{*}{ Variable } & \multirow[t]{2}{*}{ B } & \multirow[t]{2}{*}{ SE } & \multirow[t]{2}{*}{$p$} & \multirow[t]{2}{*}{ OR } & \multicolumn{2}{|c|}{$95 \% \mathrm{Cl}$ for OR } \\
\hline & & & & & Lower & Upper \\
\hline Age & 0.0212 & 0.0247 & 0.3904 & 1.0215 & 0.9731 & 1.0722 \\
\hline APACHE ॥ & 0.0541 & 0.0604 & 0.3708 & 1.0555 & 0.9377 & 1.1882 \\
\hline SOFA & 0.3630 & 0.1791 & $0.0427^{*}$ & 1.4376 & 1.0120 & 2.0422 \\
\hline mtDNA & 1.0118 & 0.4385 & $0.0210^{*}$ & 2.7506 & 1.1647 & 6.4959 \\
\hline PEEP & 0.3742 & 0.3799 & 0.3247 & 1.4539 & 0.6904 & 3.0615 \\
\hline Pplat & 0.0804 & 0.1162 & 0.4887 & 1.0838 & 0.8631 & 1.3609 \\
\hline
\end{tabular}

APACHE Acute Physiology and Chronic Health Evaluation, SOFA Sequential Organ Failure Assessment, OI oxygen index, PEEP positive end expiratory pressure, CVP central venous pressure, $N E$ norepinephrine

Table 7 General characteristics of different infection sources in the ALI group

\begin{tabular}{|c|c|c|c|}
\hline Characteristics & Abdomen, $n=10$ & Pulmonary, $n=14$ & $\mathrm{p}$ \\
\hline Age, years & $62.5(54.8-78.3)$ & $65.5(60.8-69.8)$ & 0.7933 \\
\hline \multicolumn{4}{|l|}{ Sex, n (\%) } \\
\hline Male & $6(60.0)$ & $10(71.4)$ & \\
\hline Mortality, n (\%) & $3(30.0)$ & $1(7.1)$ & \\
\hline ICU stay time, days & $8.5(4.5-27.5)$ & $20.5(13.5-41.8)$ & 0.1693 \\
\hline APACHE $\|$ & $18.0(12.3-23.3)$ & $17.5(12.8-26.5)$ & 0.5804 \\
\hline SOFA & $8.5(6.3-10.0)$ & $8.5(6.3-11.0)$ & 0.9829 \\
\hline $\begin{array}{l}\text { mtDNA, log copies/ } \\
\mu \mathrm{L}\end{array}$ & $5.14(4.39-5.87)$ & $4.79(2.52-5.54)$ & $0.0492^{*}$ \\
\hline $\mathrm{Ol}, \mathrm{mmHg}$ & $215(178-243)$ & $256(230-271)$ & $0.0369^{*}$ \\
\hline PEEP, $\mathrm{cmH} 2 \mathrm{O}$ & $7.0(6.0-8.0)$ & $5.0(5.0-6.0)$ & 0.1096 \\
\hline Pplat, $\mathrm{cmH} 2 \mathrm{O}$ & $19.0(17.0-23.0)$ & $19.0(17.5-21.5)$ & 0.8834 \\
\hline MAP, $\mathrm{mmHg}$ & 83.5 (79.5-88.5) & 85.5 (79.8-92.3) & 0.1795 \\
\hline $\mathrm{HR}$, bpm & $100.0(95.0-104.5)$ & $84.0(70.0-91.0)$ & $0.0176^{*}$ \\
\hline $\mathrm{CVP}, \mathrm{mmHg}$ & $10.0(8.5-11.0)$ & $9.0(7.8-10.3)$ & 0.6752 \\
\hline $\mathrm{NE}, \mu \mathrm{g} / \mathrm{kg} / \mathrm{min}$ & $0.23(0.06-0.73)$ & $0.11(0.05-0.18)$ & $0.0427^{*}$ \\
\hline Lactate, mmol/L & $2.0(1.0-4.4)$ & $1.5(1.1-1.7)$ & 0.0534 \\
\hline $\mathrm{PCT}, \mathrm{ng} / \mathrm{mL}$ & $8.1(3.2-93.3)$ & $2.0(0.8-6.5)$ & $0.0116^{*}$ \\
\hline $\mathrm{IL}-6, \mathrm{pg} / \mathrm{mL}$ & $572(93-1000)$ & $38(17-120)$ & $0.0006^{* *}$ \\
\hline $\mathrm{IL}-8, \mathrm{pg} / \mathrm{mL}$ & $198(121-500)$ & $108(39-192)$ & 0.0808 \\
\hline IL-10, pg/mL & $11(7-56)$ & $6(5-10)$ & 0.4274 \\
\hline TNFa, pg/mL & $25(14-35)$ & $19(13-28)$ & 0.6741 \\
\hline
\end{tabular}

ALI acute lung injury, ICU intensive care unit, APACHE Acute Physiology and Chronic Health Evaluation, SOFA Sequential Organ Failure Assessment, OI oxygen index, PEEP positive end expiratory pressure, Pplat plat pressure, $M A P$ mean arterial pressure, $H R$ heart rate, $C V P$ central venous pressure, $N E$ norepinephrine, $P C T$ procalcitonin, CRP C-reactin protein, IL-6 interleukin 6, LL-8 interleukin 8, IL-10 interleukin 10, TNFa tumor necrosis factor a

and the area under the ROC curve was 0.856 (95\% CI $0.748-0.930)$. Patients with high mtDNA levels had worse outcomes, and post hoc tests showed significant differences in 28-day survival rates (log-rank [MantelCox], 6.842; p 0.0089). Patients with high mtDNA levels experienced longer ICU stay times [21.5 (11.3-42.8) vs $6.0(4.0-12.0)$ days, $\mathrm{p}<0.0001]$, more severe organ dysfunction [SOFA score, 10.9 (7.6-14.2) vs 6.8 (3.89.7), $\mathrm{p}<0.0001]$ and worse respiratory and hemodynamic conditions.

MtDNA level (odds ratio 2.7506; 95\% CI 1.16476.4959; $\mathrm{p}$ 0.0210) was also independently associated with ALI risk. The interaction between mtDNA and innate immunity helps us to understand the mechanism of SIRS caused by injury, such as trauma or sepsis. Due to the bacterial ancestry of mitochondria, mtDNA contains unmethylated $\mathrm{CpG}$ repeats and formylated peptides that could be recognized by pattern-recognition receptors [22, 23]. mtDNA released into the cytosol or circulation could activate the TLR-9-mediated NF- $\mathrm{KB}$ pathway $[5,8]$ or interact with inflammasomes, leading to caspase- 1 activation or secretion of proinflammatory cytokines such as IL-1 $\beta$ and IL-18 $[3,24]$. Based on the molecular biology of mtDNA as a potent DAMP, circulating mtDNA may contribute to the mechanism linking distant injury to subsequent lung injury. Therefore, we hypothesized that the association between circulating mtDNA and septic ALI would be strongest in patients with extrapulmonary sepsis sources. The basic clinical characteristics of all patients showed that bloodstream and abdominal infection seemed to be more common than pulmonary infection in patients with ALI. As we expected, a higher amount of mtDNA [5.14 (4.39-5.87) vs $4.79(2.52-5.54) \log$ copies/ $\mu \mathrm{L}, \mathrm{p}$ 0.0492] was seen in patients with abdominal infection than in those with pulmonary infection. The OI of abdominal infection patients was even worse than that of pulmonary infection patients [215 (178-243) vs $256(230-271) \mathrm{mmHg}$, p 0.0369]. Consistent with our hypothesis, higher levels of inflammatory biomarkers such as PCT and IL- 6 and higher HR and NE doses all indicated a more severe inflammatory response in abdominal infection.

There are still several limitations in our study. This is a single center study, which may limit generalizability. A larger sample size or multicenter study and subgroup analysis, especially among patients with specific 
infectious sources, may help. Second, an increasing number of studies have suggested that mtDNA copy numbers may vary among different disease courses [21, 25]. Our study collected only blood samples within $24 \mathrm{~h}$ after admission, and a dynamic view of mtDNA copy number may be significant and worthy of further study.

\section{Conclusions}

Plasma mtDNA levels within $24 \mathrm{~h}$ after admission were associated with ALI incidence and mortality in septic patients in our study. The association was stronger in patients with extrapulmonary infection sources than in those with pulmonary infection.

\section{Abbreviations}

mtDNA: Mitochondrial DNA; ALI: Acute lung injury; RV: Right ventricular: DAMP: Damage-associated molecular pattern; TLR-9: Toll-like receptor 9; SIRS: Systemic inflammatory response syndrome; PUMCH: Peking Union Medical College Hospital; ICU: Intensive care unit; SOFA: Sequential Organ Failure Assessment; Ol: Oxygen index; ASE: American Society of Echocardiography; TAPSE: Tricuspid annular plane systolic excursion; APACHE: Acute Physiology and Chronic Health Evaluation; PCR: Polymerase chain reaction; ROC: Receiver operating characteristic; Pplat: Plat pressure; PEEP: Positive end expiratory pressure; CVP: Central venous pressure; MAP: Mean arterial pressure; HR: Heart rate; NE: Norepinephrine; PCT: Procalcitonin; CRP: C-reactin protein; IL-6: Interleukin 6; IL-8: Interleukin 8; IL-10: Interleukin 10; TNFa: Tumor necrosis factor a; ARDS: Acute Respiratory Distress Syndrome; PMNs: Polymorphonuclear neutrophils.

\section{Acknowledgements}

This manuscript has been edited and proofread by American Journal Experts.

\section{Authors' contributions}

LDW designed the experiment. MJY drafted and revise the manuscript. LDK assembled input data and helped to draft the manuscript, ZHM participated in the design of the study. WXT conceived of the study, participated in its design and coordination. All authors have read the manuscript and approved of the version to be published.

\section{Funding}

This work was supported by grants from the Natural Science Foundation of Beijing Municipality (No. 7194306).

\section{Availability of data and materials}

The datasets supporting the conclusions of the current study are available from the corresponding author upon reasonable request. Please contact the corresponding author if you would like to request the dataset.

\section{Ethics approval and consent to participate}

This study was approved by the ethics committee of Peking Union Medical College Hospital, Beijing, China (Approval No. JS-1985). Written informed consent was obtained from the next of kin of each patient.

\section{Consent for publication}

Not applicable.

\section{Competing interest}

The authors declare that they have no conflict of interest.

Received: 1 November 2020 Accepted: 12 February 2021

Published online: 25 February 2021

\section{References}

1. Harrington JS, Huh JW, Schenck EJ, Nakahira K, Siempos II, Choi AMK. Circulating mitochondrial DNA as predictor of mortality in critically III patients: a systematic review of clinical studies. Chest. 2019:156(6):1120-36.

2. Nakahira K, Hisata S, Choi AM. The Roles of Mitochondrial damageassociated molecular patterns in diseases. Antioxid Redox Signal. 2015;23(17):1329-50.

3. Shimada K, Crother TR, Karlin J, Dagvadorj J, Chiba N, Chen S, et al. Oxidized mitochondrial DNA activates the NLRP3 inflammasome during apoptosis. Immunity. 2012;36(3):401-14.

4. Zhang $Q$, Raoof M, Chen Y, Sumi Y, Sursal T, Junger W, et al. Circulating mitochondrial DAMPs cause inflammatory responses to injury. Nature. 2010:464(7285):104-7.

5. West AP, Shadel GS. Mitochondrial DNA in innate immune responses and inflammatory pathology. Nat Rev Immunol. 2017;17(6):363-75.

6. Nakahira K, Kyung SY, Rogers AJ, Gazourian L, Youn S, Massaro AF, et al. Circulating mitochondrial DNA in patients in the ICU as a marker of mortality: derivation and validation. PLoS Med. 2013;10(12):e1001577 (discussion e)

7. Timmermans K, Kox M, Scheffer GJ, Pickkers P. Plasma nuclear and mitochondrial DNA levels, and markers of inflammation, shock, and organ damage in patients with septic shock. Shock (Augusta, Ga). 2016:45(6):607-12.

8. Krychtiuk KA, Ruhittel S, Hohensinner PJ, Koller L, Kaun C, Lenz M, et al. Mitochondrial DNA and toll-like receptor-9 are associated with mortality in critically III patients. Crit Care Med. 2015;43(12):2633-41.

9. Omura T, Kushimoto S, Yamanouchi S, Kudo D, Miyagawa N. Highmobility group box 1 is associated with neurological outcome in patients with post-cardiac arrest syndrome after out-of-hospital cardiac arrest. J Intensive Care. 2016:4:37.

10. Zeng Z, Li D, Liu F, Zhou C, Shao Q, Ding C, et al. Mitochondrial DNA plays an important role in lung injury induced by sepsis. J Cell Biochem. 2018;120:8547-60.

11. Sun S, Sursal T, Adibnia Y, Zhao C, Zheng Y, Li H, et al. Mitochondrial DAMPs increase endothelial permeability through neutrophil dependent and independent pathways. PLoS ONE. 2013;8(3):e59989.

12. Singer $M$, Deutschman CS, Seymour CW, Shankar-Hari M, Annane D, Bauer $M$, et al. The third international consensus definitions for sepsis and septic shock (sepsis-3). JAMA. 2016;315(8):801-10.

13. Bernard GR, Artigas A, Brigham KL, Carlet J, Falke K, Hudson L, et al. The American-European Consensus Conference on ARDS. Definitions, mechanisms, relevant outcomes, and clinical trial coordination. Am J Respir Crit Care Med. 1994;149(3 Pt 1):818-24.

14. Hotz MJ, Qing D, Shashaty MGS, Zhang P, Faust H, Sondheimer N, et al. Red blood cells homeostatically bind mitochondrial DNA through TLR9 to maintain quiescence and to prevent lung injury. Am J Respir Crit Care Med. 2018;197(4):470-80.

15. Rudski LG, Lai WW, Afilalo J, Hua L, Handschumacher MD, Chandrasekaran K, et al. Guidelines for the echocardiographic assessment of the right heart in adults: a report from the American Society of Echocardiography endorsed by the European Association of Echocardiography, a registered branch of the European Society of Cardiology, and the Canadian Society of Echocardiography. J Am Soc Echocardiogr. 2010;23(7):685-713 ((quiz 86-88)).

16. Harrington JS, Choi AMK, Nakahira K. Mitochondrial DNA in Sepsis. Curr Opin Crit care. 2017;23(4):284-90.

17. Di Caro V, Walko TD 3rd, Bola RA, Hong JD, Pang D, Hsue V, et al. Plasma mitochondrial DNA — a novel DAMP in pediatric sepsis. Shock (Augusta, Ga). 2016:45(5):506-11.

18. Simmons JD, Lee YL, Mulekar S, Kuck JL, Brevard SB, Gonzalez RP, et al. Elevated levels of plasma mitochondrial DNA DAMPs are linked to clinical outcome in severely injured human subjects. Ann Surg. 2013;258(4):591-6 ((discussion 6-8))

19. Zhang JZ, Wang J, Qu WC, Wang XW, Liu Z, Ren JX, et al. Plasma mitochondrial DNA levels were independently associated with lung injury in elderly hip fracture patients. Injury. 2017:48(2):454-9.

20. Gan L, Chen X, Sun T, Li Q, Zhang R, Zhang J, et al. Significance of serum mtDNA concentration in lung injury induced by hip fracture. Shock (Augusta, Ga). 2015;44(1):52-7. 
21. Faust HE, Reilly JP, Anderson BJ, Ittner CAG, Forker CM, Zhang P, et al. Plasma mitochondrial DNA levels are associated with ARDS in trauma and sepsis patients. Chest. 2020;157(1):67-76.

22. Gray MW, Burger G, Lang BF. Mitochondrial evolution. Science. 1999;283(5407):1476-81.

23. Cardon LR, Burge C, Clayton DA, Karlin S. Pervasive CpG suppression in animal mitochondrial genomes. Proc Natl Acad Sci U S A. 1994;91(9):3799-803.

24. Nakahira K, Haspel JA, Rathinam VA, Lee SJ, Dolinay T, Lam HC, et al. Autophagy proteins regulate innate immune responses by inhibiting the release of mitochondrial DNA mediated by the NALP3 inflammasome. Nat Immunol. 2011;12(3):222-30.
25. Yang Y, Yang J, Yu B, Li L, Luo L, Wu F, et al. Association between circulating mononuclear cell mitochondrial DNA copy number and in-hospital mortality in septic patients: A prospective observational study based on the Sepsis-3 definition. PLoS ONE. 2019;14(2):e0212808.

\section{Publisher's Note}

Springer Nature remains neutral with regard to jurisdictional claims in published maps and institutional affiliations.
Ready to submit your research? Choose BMC and benefit from:

- fast, convenient online submission

- thorough peer review by experienced researchers in your field

- rapid publication on acceptance

- support for research data, including large and complex data types

- gold Open Access which fosters wider collaboration and increased citations

- maximum visibility for your research: over $100 \mathrm{M}$ website views per year

At BMC, research is always in progress.

Learn more biomedcentral.com/submissions 